\title{
Is remote work affecting health workers' sleep?
}

\author{
Cite as: CMAJ 2021 May 25;193:E780-1. doi: 10.1503/cmaj.1095941
}

Posted on cmajnews.com on May 7, 2021

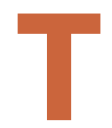

he stresses of the COVID-19 pandemic appear to be driving an increase in sleep problems among health workers. But according to a recent study published in the Journal of Clinical Sleep Medicine, those working remotely may fare worse than their peers providing care in person.

Researchers from Augusta University in Georgia surveyed more than 2300 physicians, nurses and other clinicians in an academic health system about their sleep before and during the pandemic. Of the 573 respondents with complete sleep data, more than three in five (64\%) met criteria for insomnia disorder during the pandemic, compared with more than two in five before (44.5\%).

Participants who met the criteria for insomnia had the opportunity to sleep but had trouble falling asleep or staying asleep. They also reported daytime symptoms including fatigue, difficulty concentrating, mood problems and reduced motivation. The study did not look at sleep deprivation caused by external factors such as working overnight shifts.

According to study lead Dr. Vaughn McCall, a "shockingly high" number of health workers who previously did not have sleep problems (43.4\%) newly developed insomnia during the pandemic. Women and older health workers were more likely to develop insomnia, as were those who spent less time providing care in person.

McCall said his team did not anticipate the link between insomnia and remote work and did not ask enough questions to understand the finding. "I would have thought that more time spent in direct patient care would have led to more insomnia, and yet it was the reverse," he said. "My interpretation is that working

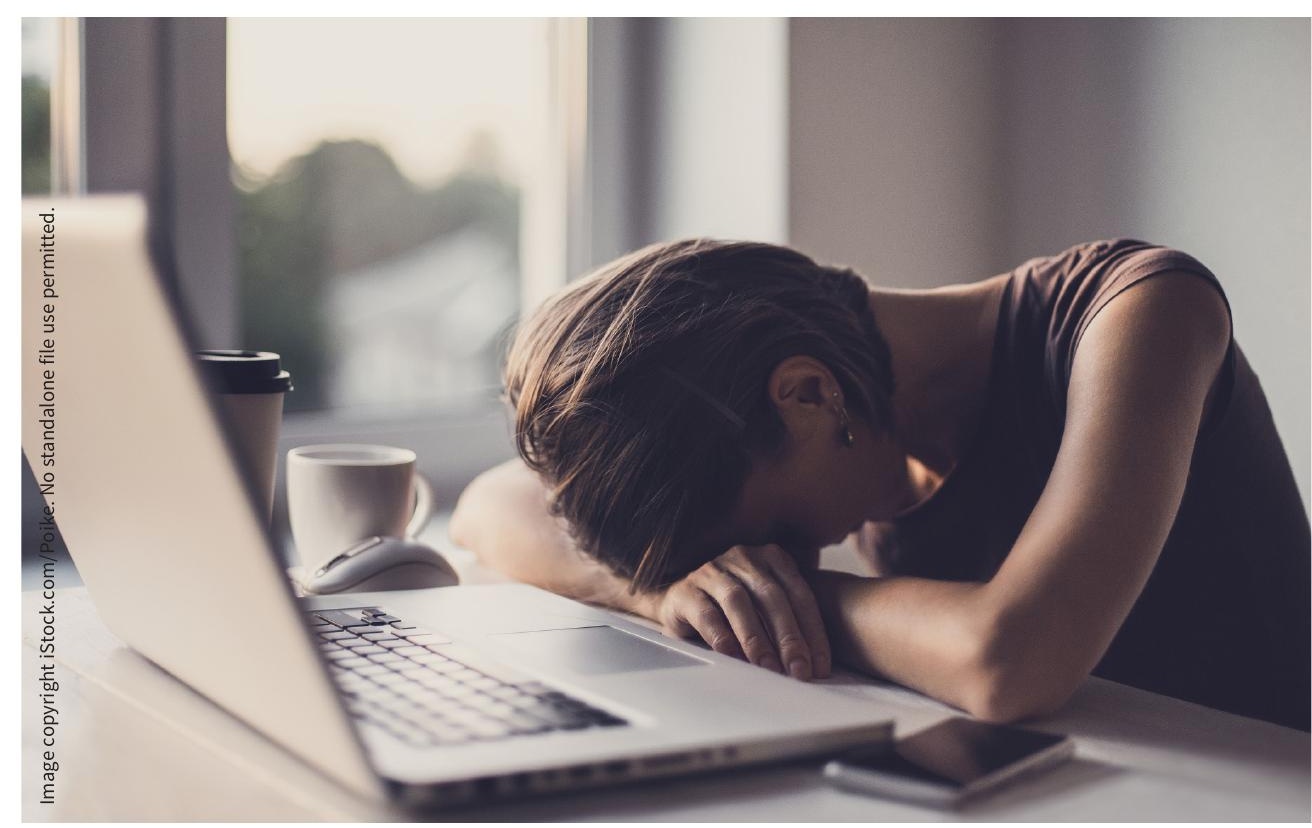

American health workers who spent less time in person with patients were more likely to develop insomnia during the pandemic.

from home is not all that it's cracked up to be."

For health workers with children, the added pressures of childcare and overseeing online education while working from home may have contributed to worse sleep, McCall said.

The study had a modest sample size and people with sleep problems may have been more likely to participate. However, the authors said their findings speak to a "need for awareness" about the impact of the pandemic on health workers' wellbeing, even if they're not on the frontline.

People typically lose sleep over timelimited stressors, and once those stressors resolve their sleep improves, said McCall. But, if the stress is prolonged as it has been during the pandemic, acute insomnia can become chronic.
Several other studies have reported increased sleep disturbances and insomnia among physicians and nurses during the pandemic. And according to a University of Ottawa meta-analysis, health workers have also reported higher rates of insomnia than the general population, consistent with trends seen during previous epidemics.

McCall and his colleagues noted that the combination of insomnia and anxiety over COVID-19 represents a "potent risk for suicidal ideation."

Poor sleep may also put front-line workers at greater risk of COVID-19. In one study published in BMJ Nutrition, Prevention \& Health, American researchers found that health workers who reported multiple sleep problems had nearly twice the risk of COVID-19. In another recent study in Thorax, British researchers found that 
people who worked irregular night shifts were three times more likely to be hospitalized for COVID-19.

Research on remote work and sleep is relatively limited. However, a 2017 paper by the United Nations International Labour Organization found that $42 \%$ of remote workers wake repeatedly during the night, compared to $29 \%$ of those working away from home.

While the pandemic has undoubtedly made life more stressful, other factors may also be contributing to poor sleep, said Dr. Colleen Carney, director of the sleep and depression laboratory at Ryerson University.

For example, many health workers have experienced changes in their work schedules which can disrupt circadian functioning that regulates sleep. Working longer hours indoors and working from home may reduce exposure to sunlight, which is vital for maintaining regular circadian rhythms, Carney explained.

"It's the reason why everyone is walking around in a fatigued state. Our circadian system is dysregulated, we're not getting enough light during the day, and then add in all the psychological stressors."

Carney recommends that people working from home get a daily dose of sunshine and exercise, and get in and out of bed on a regular schedule. Naps are not recommended for insomnia, but Carney noted they may help alleviate sleepiness on the job for shift workers.

Medication and cognitive behavioural therapy may also be helpful, especially given that "many healthcare workers have very little control over their environment right now," Carney said. "Talk to people to process the stuff that's going on at work. Don't normalize what's going on... because everyone needs support."

Diana Duong, CMAJ

Content licence: This is an Open Access article distributed in accordance with the terms of the Creative Commons Attribution (CC BY-NC-ND 4.0) licence, which permits use, distribution and reproduction in any medium, provided that the original publication is properly cited, the use is noncommercial (i.e., research or educational use), and no modifications or adaptations are made. See: https://creativecommons.org/ licenses/by-nc-nd/4.0/ 